\title{
The $g \lg X$ gene product of Corynebacterium glutamicum is required for glycogen degradation and for fast adaptation to hyperosmotic stress
}

\author{
Correspondence \\ Bernhard J. Eikmanns \\ bernhard.eikmanns@uni-ulm.de
}

Received 13 December 2006

Revised 25 January 2007

Accepted 26 February 2007

\author{
Gerd M. Seibold and Bernhard J. Eikmanns \\ Institute of Microbiology and Biotechnology, University of Ulm, D-89069 Ulm, Germany
}

\begin{abstract}
Corynebacterium glutamicum cells growing in medium containing sugars accumulate glycogen in the early exponential-growth phase, and start to degrade this polymer at entry into the stationary phase. In a first attempt to investigate glycogen degradation, the C. glutamicum glgX gene, which encodes a protein with $46 \%$ identity to the isoamylase-type debranching enzyme of Escherichia coli, was analysed, expressed and inactivated. The purified C. glutamicum gene product showed debranching activity towards glycogen, amylopectin and starch. Chromosomal inactivation of $g / g X$ in C. glutamicum wild-type led to slower growth and to a higher intracellular glycogen pool throughout growth, when compared to those in the parental strain. This result suggests that glycogen synthesis and degradation occur simultaneously in C. glutamicum. When exposed to hyperosmotic shock, C. glutamicum rapidly degrades glycogen, and at the same time, synthesizes the osmoprotectant trehalose. The glgX mutant, however, synthesized only minor amounts of trehalose throughout cultivation, and its growth ceased after hyperosmotic shock. Taken together, the results indicate that the $g \lg X$ gene product is essential for glycogen degradation in C. glutamicum, that glycogen is constantly recycled in C. glutamicum, and that it serves as a carbon store for trehalose synthesis via the TreYZ pathway after hyperosmotic shock.
\end{abstract}

\section{INTRODUCTION}

Corynebacterium glutamicum is an aerobic, Gram-positive soil bacterium well known for the industrial production of amino acids such as L-glutamate and L-lysine (Eggeling \& Bott, 2005). Moreover, this organism has gained interest as a model organism for the group of mycolic acid-containing actinomycetes, which includes several medically important species of the genus Mycobacterium (Stackebrandt et al., 1997). C. glutamicum grows on a variety of substrates, including sugars, organic acids and alcohols (Liebl, 2005). During cultivation of C. glutamicum in media containing sugars, we have recently observed synthesis and degradation of intracellular glycogen (Seibold et al., 2007). Glycogen consists of $\alpha-1,4-\alpha-1,6$-linked glucose residues (Manners, 1991), and serves as a major reserve polysaccharide in eukaryotes and in a variety of bacteria (Ball \& Morell, 2003; Iglesias \& Preiss, 1992). Although there have been some investigations of enzymes or genes involved in glycogen synthesis and degradation in other bacteria (Buschiazzo et al., 2004; Horcajada et al., 2006; Yeo \& Chater, 2005), most studies have been done with Escherichia coli (Ballicora et al., 2003; Preiss, 1996). In this organism, glycogen is synthesized in a two-step process. First, a linear $\alpha$-1,4-glycosylglucan is formed from glucose

Abbreviations: dw, dry weight; His-tag, hexahistidyl tag; WT, wild-type. 1-phosphate subunits by ADP-glucose pyrophosphorylase and glycogen synthase (Ballicora et al., 2003; Preiss, 1984). The second step, i.e. the introduction of $\alpha-1,6$-glycosidiclinkages in the polymer, is catalysed by the glycogenbranching enzyme (Abad et al., 2002). The genes encoding the three glycogen-synthesizing enzymes are located in a cluster together with genes encoding enzymes involved in degradation of glycogen (Romeo et al., 1988). Glycogen degradation in E. coli is accomplished by the interplay of four enzymes (Fig. 1). Glycogen phosphorylase forms glucose 1-phosphate and $\alpha$-1,6-branched dextrins (AlonsoCasajús et al., 2006). These phosphorylase-limit dextrins serve as substrates for the glycogen-debranching enzyme (Dauvillée et al., 2005). After debranching, the resulting linear maltodextrins serve as substrates for maltodextrin phosphorylase, which forms glucose 1-phosphate, and for maltodextrin glucosidase, which forms glucose (Dippel et al., 2005). None of these enzymes has been experimentally identified in C. glutamicum, although two genes in the genome (cg1479 and cg2289) have been annotated as glycogen phosphorylase genes, and a further gene (cg2310) has been annotated as $g l g X$ and putatively encodes a glycogen-debranching enzyme (Kalinowski et al., 2003). In contrast to E. coli, C. glutamicum degrades glycogen formed in the early exponential growth phase in the lateexponential-growth phase, i.e. before complete consumption of the sugar substrate (Seibold et al., 2007). Therefore, 


\section{Glycogen}

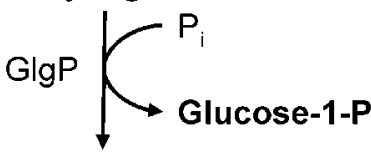

Phosphorylase-limit dextrin

( $\alpha-1,6$-branched)

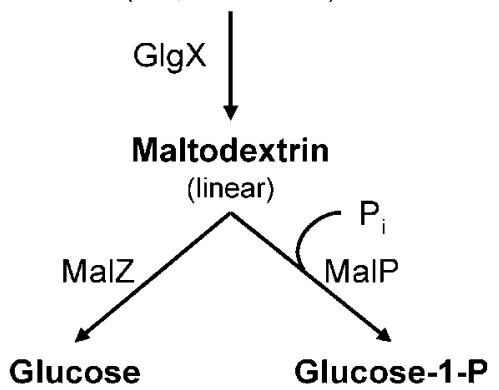

Fig. 1. Schematic diagram of glycogen degradation in E. coli. Abbreviations: GlgP, glycogen phosphorylase; GlgX, debranching enzyme; Glucose-1-P, glucose 1-phosphate; MalP, maltodextrin phosphorylase; MalZ, maltodextrin glucosidase.

it seems unlikely that glycogen in this organism serves as a long-term carbon storage compound, and in fact, glycogen metabolism in C. glutamicum has previously been suggested to be important for trehalose synthesis (Padilla et al., 2004; Seibold et al., 2007; Tzvetkov et al., 2003; Wolf et al., 2003). It has been shown that linear maltodextrins (formed as intermediates during glycogen synthesis and degradation) serve as substrates for the two-step trehalose biosynthetic pathway via maltooligosyltrehalose synthase (TreY) and maltooligosyltrehalose trehalohydrolase (TreZ) (Carpinelli et al., 2006; De Smet et al., 2000). Since trehalose in C. glutamicum acts as a cell-wall component (Tropis et al., 2005) and as a compatible solute under hyperosmotic and nitrogen-limitation conditions (Wolf et al., 2003), glycogen degradation and its control might be important for the cell-wall composition and for the response to osmotic stress in this organism.

We show here that the $g \lg X$ gene (cg2310) of C. glutamicum in fact encodes a functional glycogen-debranching enzyme. Furthermore, we investigated the role of this enzyme for growth of, and for the intracellular glycogen concentration in C. glutamicum. Due to the supposed role of glycogen degradation in trehalose formation, we further examined the role of the glycogen-debranching enzyme for growth after a shift to hyperosmotic conditions.

\section{METHODS}

Bacterial strains, plasmids and culture conditions. The bacteria used in this study were E. coli DH5 $\alpha$ (Hanahan, 1983), E. coli ER2566 (New England Biolabs) and C. glutamicum wild-type (WT) (strain ATCC 13032; American Type Culture Collection). Vector pET28a (Novagen) was used for the generation of the His-tagged GlgX. E. coli and all pre-cultures of C. glutamicum were grown aerobically in TY complex medium (Sambrook \& Russel, 2001) at 37 and $30{ }^{\circ} \mathrm{C}$, respectively, as $60 \mathrm{ml}$ cultures in $500 \mathrm{ml}$ baffled Erlenmeyer flasks on a rotary shaker at 120 r.p.m. For the main cultures of C. glutamicum, cells of an overnight pre-culture were washed with $0.9 \%(\mathrm{w} / \mathrm{v}) \mathrm{NaCl}$, and then inoculated into CgXII minimal medium (Eikmanns et al., 1991) containing glucose at the concentrations indicated in Results. C. glutamicum was grown aerobically at $30{ }^{\circ} \mathrm{C}$ as $50 \mathrm{ml}$ cultures in $500 \mathrm{ml}$ baffled Erlenmeyer flasks on a rotary shaker at 120 r.p.m., or as $250 \mathrm{ml}$ cultures in a parallel fermentation system (Fedbatch-pro system; DASGIP) as described previously (Seibold et al., 2007). In this system the $\mathrm{pH}$ was maintained at 7.0, and dissolved oxygen was adjusted to $30 \%$ saturation. Growth of E. coli and C. glutamicum was followed by measuring $\mathrm{OD}_{600}$.

DNA isolation, transfer and manipulations. Standard procedures were employed for plasmid isolation, cloning and transformation of E. coli $\mathrm{DH} 5 \alpha$, as well as for electrophoresis (Sambrook \& Russel, 2001). Isolation of C. glutamicum chromosomal DNA, transformation of C. glutamicum, and selection of the transformants were performed as described by Eikmanns et al. (1994).

All enzymes used were obtained from MBI-Fermentas and used according to the instructions of the manufacturer. PCR experiments were performed in a Biometra personal cycler (Biotron). dNTPs were obtained from MBI-Fermentas, and oligonucleotide primers from MWG-Biotech. Cycling times and temperatures were chosen according to fragment length and primer constitution. PCR products were purified using the Nucleospin Extract II kit (Macherey-Nagel).

Construction of C. glutamicum WT-ImX. Inactivation of the chromosomal $\mathrm{glgX}$ gene in C. glutamicum was performed as described previously for the inactivation of the $g \lg C$ gene (Seibold et al., 2007), using pK19mobsacB-InsglgX. This plasmid was constructed by PCR amplification of a DNA fragment covering nt 439-1604 of the annotated $g l g X$ gene, including the EcoRI restriction sites at positions 459 and 1594, using primers IMglgXFor (5'-GCAGATAACAACGAGACTC-3') and IMglgXRev (5'-GAGGAACCAGTTAGTCGGG-3'). After digestion of the PCR product with EcoRI, the fragments were separated on an agarose gel, and an 1136 bp internal fragment of $g \lg X$ was eluted from the gel using the Nucleospin Extract II kit. This fragment was ligated into the EcoRI-restricted $\mathrm{pK} 19$ mobsacB, and the resulting vector pK19mobsacB-InsglgX was transformed into E. coli. After isolation of the recombinant plasmid, it was electroporated into C. glutamicum WT. Integration of pK19mobsacB-InsglgX at the genomic glgX locus in C. glutamicum was confirmed by Southern blot hybridization, using a $g l g X$-specific $535 \mathrm{bp}$ fragment (covering nt 739-1274 of the $g l g X$ gene), amplified from chromosomal DNA of C. glutamicum WT using primers GlgXInsFor (5'-ACGCTGCCAACAAGAACC-3') and GlgXInsRev (5'-ACCACGCCAGAAATCACG-3'). Labelling, hybridization, washing and detection were conducted using the non-radioactive DNA labelling and detection kit, according to the instructions from Roche Diagnostics. The labelled probe was hybridized to BamHI-restricted and size-fractionated chromosomal DNA from C. glutamicum WT and the integration mutant WT-ImX. The hybridization resulted in one signal of $\sim 5 \mathrm{~kb}$ from C. glutamicum WT and two signals of $\sim 4.2$ and $\sim 7.8 \mathrm{~kb}$ from C. glutamicum WT-ImX. These sizes were expected for the WT strain and the $g l g X$ integration mutant, respectively.

Construction of E. coli ER2566 (pET28a-glgXHIS). Vector pET28a was used for the synthesis of the hexahistidyl (His-)tagged $\mathrm{GlgX}$ fusion protein (GlgX-His). The $g \lg X$ gene was amplified from chromosomal DNA of C. glutamicum WT by PCR with primers GlgXHisFor (5'-CATGCCATGGCATCAACGATTGAGCACTCGT3', NcoI site underlined) and GlgXHisRev (5'-CGCCTCGAGCTTCTCTTCTTCGTCCGCTTC-3', Xhol site underlined). The PCR 
product was restricted with $\mathrm{NcoI}$ and $\mathrm{XhoI}$, ligated into the $\mathrm{NcoI} /$ XhoI-restricted plasmid pET28a, and transformed into E. coli ER2566. The nucleotide sequence of the $g l g X$ fragment in pET28a-glgX[HIS] was verified by sequence analysis (MWG Biotech).

Protein analysis. Protein concentrations were determined using the bicinchoninic acid protein assay reagent kit (Pierce), with BSA as the standard. SDS-PAGE was performed according to Laemmli (1970). Western blot experiments for detection of the His-tagged GlgX protein were perfomed using His-tag mAbs and LumiBlot reagents from Novagen.

Purification of the GlgX-His protein. The crude extract for GlgXHis purification was obtained as described previously for purification of RamA (Cramer et al., 2006). The crude extract was applied to a HisTrap FF crude column (GE Healthcare) equilibrated with binding buffer ( $20 \mathrm{mM}$ sodium phosphate, $0.5 \mathrm{M} \mathrm{NaCl}, 30 \mathrm{mM}$ imidazole, $\mathrm{pH}$ 7.5). The stepwise elution of proteins with imidazole was performed and monitored with an Äkta Purifier system (GE Healthcare). The first washing buffer to elute non-specifically bound proteins contained $75 \mathrm{mM}$ imidazole, $0.5 \mathrm{M} \mathrm{NaCl}$, and $20 \mathrm{mM}$ sodium phosphate, $\mathrm{pH}$ 7.5. GlgX-His was eluted with buffer containing $250 \mathrm{mM}$ imidazole. For further purification and removal of imidazole, the fraction was applied to a HiLoad 16/60 Superdex 200 prep grade gel-filtration column (GE Healthcare) equilibrated with $40 \mathrm{mM}$ acetate buffer, $\mathrm{pH}$ 5.2. Elution was performed with a constant flow $\left(1 \mathrm{ml} \mathrm{min}{ }^{-1}\right)$. The fractions were collected and screened by Western blot analysis. For molecular mass determination, the retention volume of GlgX-His was compared to that of standard proteins (HMW Calibration kit, GE Healthcare).

Glycogen degradation/debranching activity of purified GIgXHis enzyme. For analysis of the degradation activity of the purified GlgX-His on glycogen, amylopectin and soluble starch, $7.5 \mu \mathrm{g}$ protein was added to a solution of the respective polysaccharide $\left(10 \mathrm{mg} \mathrm{ml}^{-1}\right.$ in $40 \mathrm{mM}$ acetate buffer, $\mathrm{pH} 5.3$ ). After incubation at $30{ }^{\circ} \mathrm{C}$, the $\mathrm{GlgX}$ His enzyme was inactivated by incubation at $95{ }^{\circ} \mathrm{C}$ for $10 \mathrm{~min}$. The amount of sugars released after treatment with GlgX-His compared to untreated polysaccharide was determined essentially as described by Miller (1959). Dinitrosalicylic acid solution $(0.4 \mathrm{ml}$, containing $1 \%$, $\mathrm{w} / \mathrm{v}$, dinitrosalicylic acid, $20 \%$, w/v, NaOH, and $30 \%$, w/v, potassium sodium tartrate) was added to $0.4 \mathrm{ml}$ sample. Subsequently, the samples were boiled for $5 \mathrm{~min}$ and centrifuged for $5 \mathrm{~min}$ at $13000 \mathrm{~g}$ to precipitate insoluble particles. The supernatant $(200 \mu \mathrm{l})$ was diluted with $800 \mu \mathrm{l}$ water and $A_{546}$ was recorded. The amount of reducing sugars formed was determined by comparison with a maltose standard.

The debranching activity of GlgX-His with glycogen, amylopectin and soluble starch as substrates was tested qualitatively by following changes in the spectrum of the iodine-carbohydrate complex in a spectrophotometer between 400 and $600 \mathrm{~nm}$ (Jeanningros et al., 1976). The respective carbohydrate $\left(5 \mathrm{mg} \mathrm{ml}^{-1}\right)$ was dissolved in $40 \mathrm{mM}$ acetate buffer, $\mathrm{pH}$ 5.3. After addition of $5 \mu \mathrm{g}$ purified enzyme, the reaction mixture was incubated at $30^{\circ} \mathrm{C}$. At time points $0,1,2,4$ and $24 \mathrm{~h}$ after the start of incubation, samples of $50 \mu \mathrm{l}$ were taken and heat-inactivated $\left(10 \mathrm{~min}, 95{ }^{\circ} \mathrm{C}\right)$. Iodine reagent $(2.5 \mathrm{ml} ; 0.02 \%$, w/v, iodine and $0.2 \%, \mathrm{w} / \mathrm{v}$, potassium iodine in $0.05 \mathrm{M} \mathrm{HCl}$ ) was added and the absorbance spectrum of the complex formed was determined.

Debranching activity of the GlgX-His protein was also determined in combination with $\alpha$ - and $\beta$-amylases. Aliquots of the GlgX-Histreated and untreated carbohydrate solutions (see above) were incubated with $\alpha$-amylase $\left(5 \mathrm{U} \mathrm{ml}^{-1}, 2 \mathrm{~h}, 30^{\circ} \mathrm{C}\right.$, in $0.1 \mathrm{M}$ phosphate buffer, $\mathrm{pH} 6.9)$ or with $\beta$-amylase $\left(5 \mathrm{U} \mathrm{ml}^{-1}, 2 \mathrm{~h}, 30{ }^{\circ} \mathrm{C}\right.$, in $0.1 \mathrm{M}$ acetate buffer, $\mathrm{pH}$ 4.8). Afterwards, the release of reducing sugars from the substrate was measured with dinitrosalicylic acid solution, as described above.
Quantification of glucose. For analysis of the glucose concentration in the culture broth, $1 \mathrm{ml}$ culture was withdrawn and centrifuged (13000 $\mathrm{g}, 10 \mathrm{~min}, 4{ }^{\circ} \mathrm{C}$ ), and the supernatant was used for the determination. Glucose was determined enzymically using hexokinase/glucose-6-phosphate dehydrogenase (Roche Diagnostics) and spectrophotometric quantification (at $340 \mathrm{~nm}$ ) of the NADPH formed.

Analysis of intracellular glycogen and trehalose. For enzymic analysis of intracellular glycogen and trehalose, $5 \mathrm{ml}$ samples of respective cultures were harvested, and the cell extracts were prepared exactly as described previously (Seibold et al., 2007). Glycogen was determined with amyloglycosidase, as described previously (Seibold et al., 2007). For trehalose determination, each sample was divided into two $100 \mu \mathrm{l}$ aliquots (reactions $\mathrm{A}$ and $\mathrm{B}$ ), and $1.5 \mu \mathrm{l}$ trehalase (10 $\mathrm{U}$ $\mathrm{ml}^{-1}$; Sigma) was added to reaction $\mathrm{A}$, while reaction $\mathrm{B}$ remained as a reference. Both reaction $\mathrm{A}$ and reaction $\mathrm{B}$ were incubated with shaking for $2 \mathrm{~h}$ at $37^{\circ} \mathrm{C}$. The concentration of the liberated glucose was then measured in both control (-trehalase) and experimental (+trehalase) tubes, as described above. The calculations of the intracellular trehalose content in relation to the cell dry weight $(\mathrm{dw})$ were performed as described previously for the glycogen content (Seibold et al., 2007).

TLC was used to distinguish between intracellular glycogen, trehalose and other carbohydrates, which might serve as substrates for amyloglucosidase or trehalase. Sample preparation, application, separation and visualization of the carbohydrates on the TLC plate were performed as described previously (Seibold et al., 2007). Carbohydrates were identified by comparison with the migration of known standards, i.e. glucose, trehalose, maltose, maltotriose, maltoheptaose and glycogen (obtained from Sigma).

Computational analysis. Database searches and alignments were carried out by using BLAST (Altschul et al., 1990) and CLUSTALW (Thompson et al., 1994). The TrEMBL accession numbers for (putative) $\mathrm{GlgX}$ protein sequences and the corresponding genes annotated as $g l g X$ are: C. glutamicum, Q8NNTO, cg2310; Corynebacterium diphtheriae, Q6NGF3, DIP1572; Corynebacterium efficiens, Q8FNY3, CE2010; Corynebacterium jeikeium, Q4JW65, jk0780, Mycobacterium bovis, P0A4Y5, Mb1591c; Mycobacterium tuberculosis, P0A4Y4, MT1615; E. coli, P15067, b3431.

\section{RESULTS}

\section{Nucleotide sequence of the C. glutamicum glgX gene and analysis of the deduced GIgX amino acid sequence}

The C. glutamicum cg2310 gene has been sequenced in the course of the determination of the genome sequence, and annotated as $g l g X$ due to similarities of the deduced polypetide with the debranching enzyme of E. coli (Kalinowski et al., 2003). The C. glutamicum glgX gene has a length of $2511 \mathrm{bp}$, and the predicted gene product consists of 836 aa with a molecular mass of $94334 \mathrm{Da}$. The best homology scores with probable debranching enzymes were observed with enzymes deduced from other Corynebacterineae, such as C. efficiens ( $86 \%$ identity), C. diphtheriae (80\%), C. jeikeium (72\%), M. tuberculosis $(68 \%)$ and M. bovis (68\%), while the best score with a functionally proven enzyme was that with the E. coli debranching enzyme GlgX ( $46 \%$ identity). The $g l g X$ gene 
of C. glutamicum is preceded by an ORF (cg2309) which probably encodes a TetR-like regulator, and is followed by cg2311, which putatively encodes an $S$-adenosylmethionine (SAM)-dependent methyltranferase (Kalinowski et al., 2003). A similar genomic organization can be observed in C. efficiens (Tauch et al., 2005) and C. diphtheriae (Cerdeno-Tarraga et al., 2003), whereas in C. jeikeium, the deduced $g l g X$ gene seems to form an operon with treY (Nishio et al., 2003). This is also the case in M. tuberculosis and $M$. bovis, in which the annotated $g l g X$ gene is directly followed by the genes encoding TreY and TreZ, which may form $g l g X-t r e Y-t r e Z$ operons (Fleischmann et al., 2002; Garnier et al., 2003). In C. glutamicum, $g l g X$ is located $\sim 10 \mathrm{~kb}$ apart from tre $Y$, and the tre $Y$ and tre $Z$ genes are separated by $\sim 8 \mathrm{~kb}$.

\section{Inactivation of the $g / g X$ gene and analysis of the mutant}

To study the role of the $g \lg X$ gene product (GlgX protein) in growth and glycogen degradation in C. glutamicum, the chromosomal $g \lg X$ gene was inactivated by chromosomal insertion of the vector $\mathrm{pK} 19$ mobsacB-InsglgX, resulting in strain C. glutamicum WT-ImX. During cultivation in minimal medium containing $1 \%$ glucose, growth behaviour and glycogen content of the mutant C. glutamicum WT-ImX were significantly different from those of $C$. glutamicum WT (Fig. 2). In comparison to the WT, the growth rate in the exponential-growth phase was reduced (WT, $0.37 \mathrm{~h}^{-1}$; WT-ImX, $0.26 \mathrm{~h}^{-1}$ ); the final optical density of the mutant strain was significantly lower (14.1

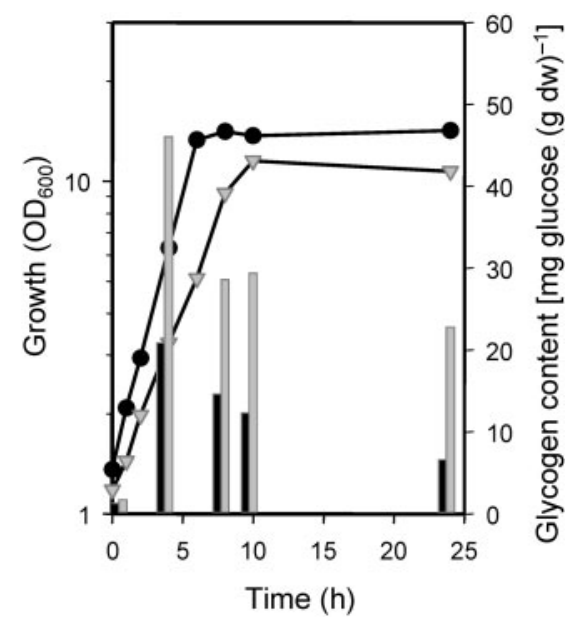

Fig. 2. Growth (lines) and glycogen content (bars) of $C$. glutamicum WT (black circles and bars) and C. glutamiucm WTImX (grey triangles and bars) during cultivation in minimal medium with $1 \%$ glucose as a carbon source. Glycogen was determined enzymically as liberated glucose after amyloglycosidase treatment (two determinations per sample). Three independent cultivations were performed, and the SD of the glycogen determinations was $<10 \%$. and 10.5, respectively), and throughout cultivation, $C$. glutamicum WT-ImX showed an increased glycogen content. Moreover, the glycogen content decreased only partially in the course of the stationary phase, leading even $16 \mathrm{~h}$ after entry into the stationary phase to a glycogen content of $22.5 \mathrm{mg}$ glucose $(\mathrm{g} \mathrm{dw})^{-1}$ (Fig. 2). In contrast, at that time point, only minor amounts of glycogen were present in C. glutamicum WT [6 $\mathrm{mg}\left(\mathrm{g} \mathrm{dw}^{-1}\right]$. The intracellular carbohydrates were additionally tested at selected time points via TLC analysis. In accordance with the enzymic measurements shown in Fig. 2, the TLC analysis revealed that almost no glycogen was present $24 \mathrm{~h}$ after inoculation in C. glutamicum WT cells, whereas glycogen was present in the mutant (Fig. 3). Analysis of the samples taken in the course of the exponential-growth phase revealed that C. glutamicum WT samples, but not the mutant samples, contained several other smaller carbohydrates in addition to glycogen, with sizes in the range of maltotetraose to maltooctaose (Fig. 3). These carbohydrates obviously are intermediates of glycogen degradation.

\section{Purification of GlgX-His and analysis of its activity}

To further analyse the function of the $g l g X$ gene and its role in glycogen degradation in C. glutamicum, we purified and characterized the respective $\mathrm{GlgX}$ protein. GlgX was synthesized as a C-terminal His-tagged fusion protein in E. coli ER2566 and purified using centrifugation, affinity

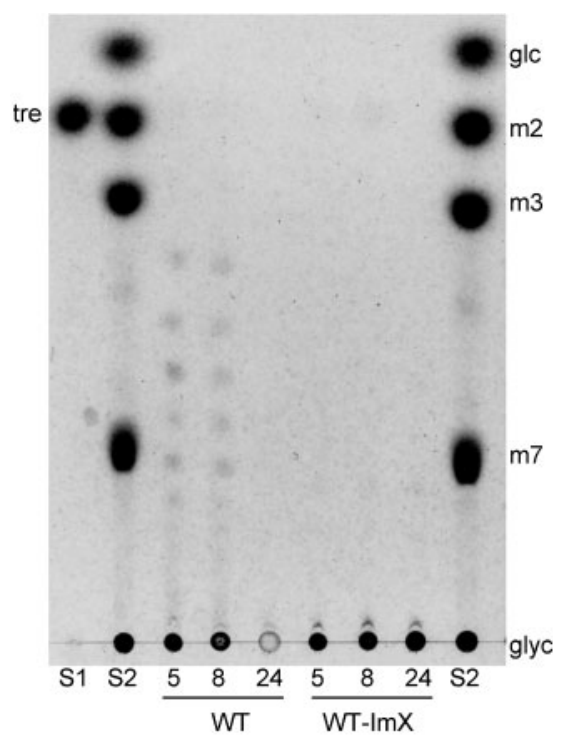

Fig. 3. TLC analysis of intracellular carbohydrates of C. glutamicum WT and C. glutamicum WT-ImX during cultivation in minimal medium with $1 \%$ glucose as a carbon source. The samples were taken at 5, 8 and $24 \mathrm{~h}$ after inoculation. Lanes S1 and S2 represent the separation of the standard sugars: standard solution S1 contained trehalose (tre), and standard solution S2 contained glucose (glc), maltose (m2), maltotriose (m3), maltoheptaose (m7) and glycogen (glyc). 
chromatography and size-exclusion chromatography. SDSPAGE analysis of the products of each purification step finally revealed a single protein of $\sim 110 \mathrm{kDa}$, which could also be detected by Western blot analysis with antibodies binding to the C-terminal His-tag (data not shown). Sizeexclusion chromatography with the GlgX-His and standard proteins revealed a native molecular mass of $417000 \mathrm{Da}$ (data not shown). This result allows the assumption that native $\mathrm{GlgX}$-His forms a homotetramer.

To determine the extent of degradation activity of $\mathrm{GlgX}$ towards glycogen, soluble starch and amylopectin, they were incubated with purified GlgX-His protein, and the number of reducing ends formed was determined with dinitrosalicylic acid. Incubation of the polysaccharides with purified GlgX-His protein for 30 min led to liberation of 0.11-0.17 milligram maltose equivalents per millilitre compared to that in untreated substrates.

Debranching activity of the purified $\mathrm{GlgX}$-His protein was assayed with glycogen, soluble starch and amylopectin as substrates by following changes in iodine colouration. A change in the absorbance maximum of the iodinecarbohydrate complex (from 471 to $477 \mathrm{~nm}$, with glycogen as a substrate), and an increase in absorption of the complex formed was observed after incubation of purified GlgX-His with each of the three substrates. Although this method does not allow quantitative determination, the result shows that GlgX-His is able to cleave $\alpha-1,6$-glycosidic linkages.

To further test for debranching activity of GlgX, glycogen, starch and amylopectin were pre-treated with GlgX-His, incubated with $\alpha$ - or $\beta$-amylase, and the number of reducing ends formed was determined with dinitrosalicylic acid. Degradation of branched polysaccharides (such as glycogen or starch) by $\alpha$ - and $\beta$-amylase is limited by $\alpha-1$ 6 -glycosidic linkages, which leads to the formation of $\alpha$ - or $\beta$-amylase-limit dextrins (Friedberg \& Rhodes, 1986; Ikawa et al., 1998). Pre-treatment of glycogen or starch with a debranching enzyme should reduce the amount of these amylase-limit dextrins formed, and increase the formation of reducing ends by $\alpha$ - and/or $\beta$-amylase. Comparing the number of reducing ends formed by the action of $\alpha$ - or $\beta$ amylase alone with the number of reducing ends in assays with $\mathrm{GlgX}$-His pre-treatment showed that, in the latter assays, up to an additional 0.42 milligrams maltose equivalents per millilitre was liberated (Fig. 4). No additional reducing ends were liberated by the action of GlgX-His on amylose, a polysaccharide consisting of $\alpha-1,4-$ linked glucose residues only (data not shown). Thus, incubation of the branched polysaccharides glycogen, starch and amylopectin with purified protein GlgX-His led to the formation of additional liberated maltose equivalents, compared to an assay with only $\alpha$ - or $\beta$ amylase-treated substrates. These results indicate that due to the action of GlgX-His, the availability of $\alpha-1,4$ glycosidic-linked glucose residues for $\alpha$ - and for $\beta$-amylase is increased. These results and the observations made with

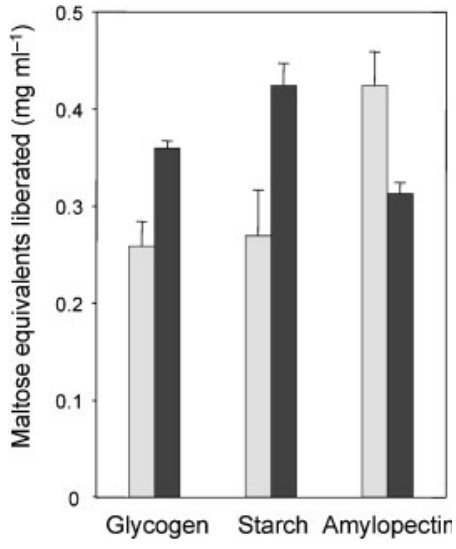

Fig. 4. Debranching activity of purified C. glutamicum GlgX-His protein. The release of maltose equivalents was measured with dinitrosalicylic acid from the substrates glycogen, starch and amylopectin. Bars represent additional reducing ends liberated by $\alpha$-amylase (light grey) or $\beta$-amylase (dark grey) after pre-treatment of the respective substrate with GlgX-His, when compared to nonpre-treated substrates. The reducing ends formed by $\alpha$-amylase without GlgX-His pretreatment correspond to $1.85 \mathrm{mg} \mathrm{ml}^{-1}$ (glycogen), $3.51 \mathrm{mg} \mathrm{ml}^{-1}$ (starch) and $2.77 \mathrm{mg} \mathrm{ml}^{-1}$ (amylopectin) maltose equivalents; those formed by $\beta$-amylase correspond to $3.26 \mathrm{mg} \mathrm{ml}^{-1}$ (glycogen), $4.65 \mathrm{mg} \mathrm{ml}^{-1}$ (starch) and $4.33 \mathrm{mg} \mathrm{ml}^{-1}$ (amylopectin) maltose equivalents.

the $g \lg X$ mutant show that $\mathrm{GlgX}$ is involved in glycogen degradation, and indicate that it represents the debranching enzyme of $C$. glutamicum.

\section{Role of the debranching enzyme GIgX for growth under hyperosmotic conditions}

Previous studies have revealed a link between glycogen and trehalose synthesis in C. glutamicum, suggesting a role of glycogen metabolism for fast adaptation to hyperosmotic growth conditions (Padilla et al., 2004; Seibold et al., 2007; Tzvetkov et al., 2003; Wolf et al., 2003). We therefore examined the effects of salt addition in the exponentialgrowth phase on growth, substrate consumption, glycogen level and intracellular trehalose formation of C. glutamicum WT, using minimal medium with $1.5 \%$ glucose as a carbon source. Addition of $750 \mathrm{mM} \mathrm{NaCl} 5.5 \mathrm{~h}$ after inoculation to the culture broth led to a decrease in growth rate from 0.35 to $0.19 \mathrm{~h}^{-1}$, and to a decrease in glucose consumption rate (Fig. 5a). Immediately after the addition of salt, the glycogen level dropped severely from 62 to $4 \mathrm{mg}$ glucose $(\mathrm{g} \mathrm{dw})^{-1}$ (Fig. 5b). In the course of further growth, however, the glycogen content rose again. Interestingly, shortly after hyperosmotic shock, the intracellular trehalose concentration was significantly higher when compared to that in the cells not treated with $\mathrm{NaCl}$ (Fig. 5c). This result suggests that, as a consequence of hyperosmotic shock, glycogen is degraded, and trehalose is formed from the degradation products of glycogen. To investigate this 

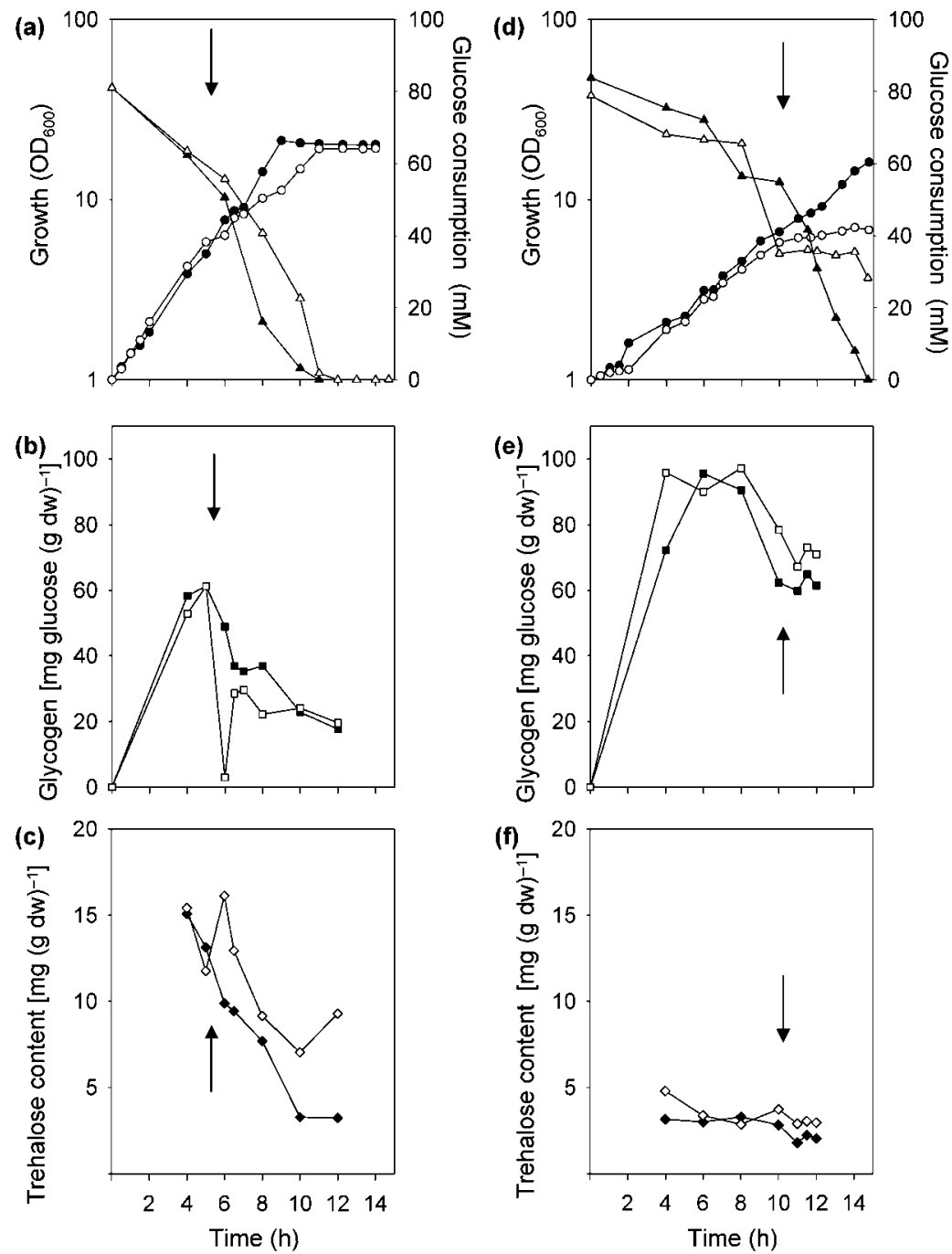
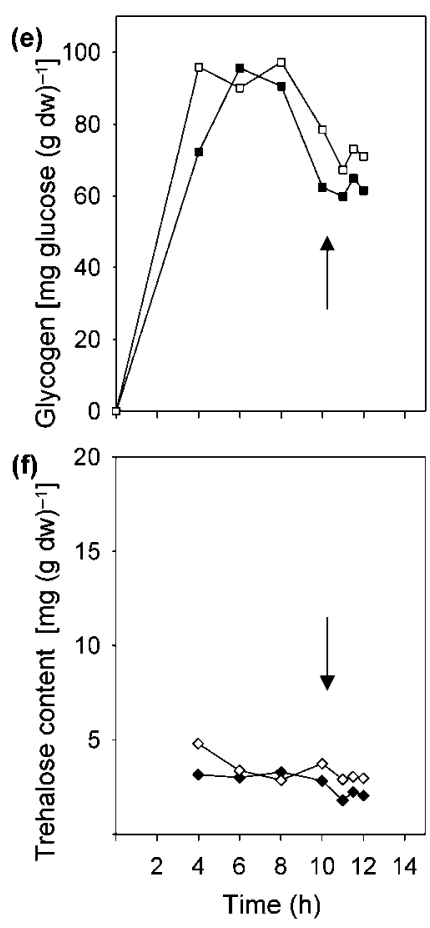

Fig. 5. Effects of hyperosmotic shock by addition of $750 \mathrm{mM} \mathrm{NaCl}$ on growth (circles), glucose consumption (triangles), glycogen content (squares) and trehalose content (diamonds) of C. glutamicum WT $(\mathrm{a}-\mathrm{c})$ and $\mathrm{C}$. glutamicum WT-ImX (d-f) during cultivation in minimal medium with $1.5 \%$ glucose as a carbon source. Solid symbols, the respective strain without salt addition; open symbols, with salt addition, at the time point indicated by the arrows. hypothesis and to study the function of $\mathrm{GlgX}$ in response to hyperosmotic conditions, we performed the same experiments with mutant C. glutamicum WT-ImX, which is unable to degrade glycogen. Addition of $750 \mathrm{mM} \mathrm{NaCl}$ at the same optical density as in the experiment with $C$. glutamicum WT immediately halted growth and glucose consumption (Fig. 5d). As expected from the results described above, more glycogen was present throughout the growth of cells of the mutant strain WT-ImX, compared to C. glutamicum WT (Fig. 5b, e). Moreover, the addition of salt to the medium did not lead to a decrease in glycogen content of the mutant strain WT-ImX (Fig. 5e). These results indicate that the $\mathrm{GlgX}$ protein is essential for glycogen degradation after hyperosmotic shock. The intracellular trehalose concentration of the $g \lg X$ mutant strain (Fig. 5f) throughout cultivation was lower than the concentration observed in the WT strain. Moreover, the addition of $\mathrm{NaCl}$ to the medium did not lead to an increase in the trehalose content (Fig. 5f).
The intracellular glycogen and trehalose content after hyperosmotic shock in C. glutamicum WT and in the mutant strains was also investigated in a separate experiment by TLC analysis. For this purpose, culture samples were taken immediately (30 s) before (at $6 \mathrm{~h}$ for the WT and $10 \mathrm{~h}$ for the $g \lg X$ mutant strain) and $30 \mathrm{~min}$ after the addition of $750 \mathrm{mM} \mathrm{NaCl}(6.5 \mathrm{~h}$ for the WT and $10.5 \mathrm{~h}$ for the mutant). Further samples from the WT and WT-ImX strain were taken at the late-stationary phase, i.e. $24 \mathrm{~h}$ after inoculation. As shown in Fig. 6, glycogen and smaller carbohydrates were present in cell extracts of $C$. glutamicum WT at the exponential-growth phase (6 and $6.5 \mathrm{~h}$ after inoculation). In the $24 \mathrm{~h}$ sample, no glycogen was detected. After the addition of salt to the medium at $6.5 \mathrm{~h}$, an additional spot with retention behaviour corresponding to that of trehalose occurred, and was still detectable in the sample taken at the stationary phase $(24 \mathrm{~h}$ after inoculation, Fig. 6). As in the TLC analysis of cell extracts from the mutant strain WT-ImX grown without 


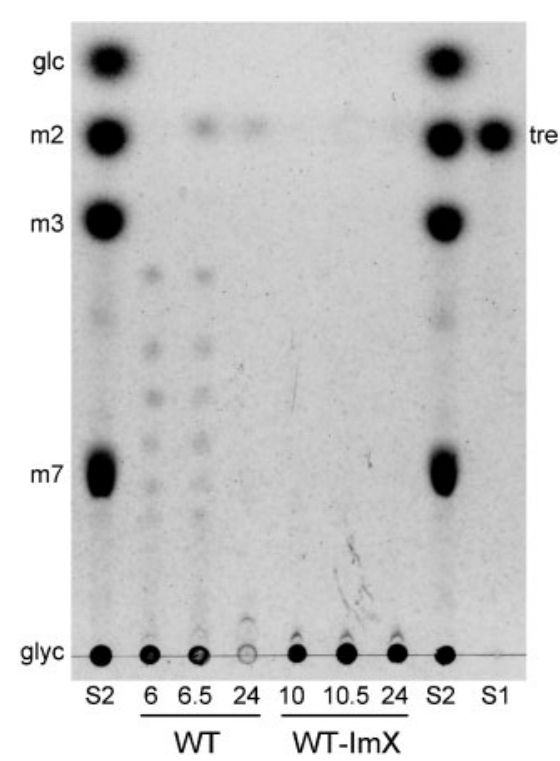

Fig. 6. TLC analysis of intracellular carbohydrates of C. glutamicum WT and C. glutamicum WT-ImX during cultivation in minimal medium with $1.5 \%$ glucose as a carbon source, before and after hyperosmotic shock. $\mathrm{NaCl}$ was added to C. glutamicum WT $6 \mathrm{~h}$ after inoculation and to C. glutamicum WT- $\operatorname{ImX} 10 \mathrm{~h}$ after inoculation. The numbers given above WT and WT-ImX indicate the time points (hours after inoculation) of sample collection. Lanes S1 and S2 represent separation of the standard sugars: standard solution S1 contained trehalose (tre), and standard solution S2 contained glucose ( $\mathrm{glc}$ ), maltose (m2), maltotriose (m3), maltoheptaose (m7) and glycogen (glyc).

addition of sodium chloride (Fig. 3), all samples of the mutant taken before ( $10 \mathrm{~h}$ after inoculation) and after salt addition (at $10.5 \mathrm{~h}$ ) contained glycogen, but no trehalose or other carbohydrate (Fig. 6). These results indicate that glycogen degradation is necessary for fast synthesis of trehalose as a compatible solute, and for survival after hyperosmotic shock.

\section{DISCUSSION}

In contrast to glycogen synthesis, glycogen degradation has not been investigated so far in C. glutamicum. We showed here that inactivation of the chromosomal $\operatorname{glg} X$ gene in $C$. glutamicum led throughout growth to a higher intracellular glycogen content when compared to that in C. glutamicum WT, and only partial degradation of glycogen (possibly due to activity of glycogen phosphorylase; see Fig. 1) at the end of the exponential-growth phase was observed with the mutant. Furthermore, we showed that the purified $g \lg X$ gene product was able to cleave $\alpha$-1,6-glycosidic linkages in glycogen, starch and amylopectin. These results indicate that the C. glutamicum $g l g X$ gene product is in fact involved in glycogen degradation, and that $g \lg X$ encodes a glycogen-debranching enzyme.
It is obvious that glycogen degradation in C. glutamicum WT takes place in the late-exponential-growth phase. However, at the beginning of the exponential-growth phase, the glycogen content of the $g l g X$ mutant strain was much higher than that of C. glutamicum WT (Fig. 2), indicating that the debranching reaction has already taken place at this growth phase, i.e. when glycogen is still being synthesized. Parallel synthesis and degradation of glycogen have also been observed in E. coli. In this organism, the key enzymes for glycogen degradation, glycogen phosphorylase and debranching enzyme, are present and active throughout growth, even when there is net glycogen accumulation (Dauvillée et al., 2005). In agreement with these observations, the genes encoding the E. coli proteins involved in glycogen degradation and synthesis $(g l g C, g l g A, g l g B, g l g P$ and $g l g X$ ) are clustered and arranged in operons (Romeo et al., 1988). However, in C. glutamicum, the glg genes or their putative homologues are obviously scattered within the genome (Seibold et al., 2007).

Polysaccharide preparations of C. glutamicum WT-ImX lacked maltodextrins (Fig. 3). This result was rather surprising, since during glycogen synthesis, the glycogen synthase should form linear glucans, which subsequently obtain $\alpha-1,6$-glycosidic-linked residues by the action of the branching enzyme GlgB. We have recently observed that GlgB of C. glutamicum acts very efficiently on maltodextrins, and is involved in the fast formation of large polysaccharides (unpublished data). If glycogen branching takes place simultaneously with glycogen debranching, an equilibrium between linear and $\alpha-1,6$-glycosidic-linked maltodextrins (thereby forming a glycogen-like structure) is established. The lack of debranching, caused by disruption of the $g \lg X$ gene, would then shift the equilibrium in the direction of branched maltodextrins. This seems to be the case in C. glutamicum WT-ImX, in which only a large glycogen-like polysaccharide is detectable, and no more maltodextrins are visible in carbohydrate preparations of cell extracts. We therefore believe that the maltodextrins observed in the WT are an additional indicator for degradation processes taking place in parallel to synthesis. This hypothesis is in agreement with a possible role of glycogen as a carbon capacitor, as has previously been suggested for Mycobacterium smegmatis (Belanger \& Hatfull, 1999). According to this model, the flow of carbon into glycolysis is modulated by the glycogen pool to prevent wasteful expenditure of resources.

For survival of the bacterial cell, it is necessary to react fast to sudden changes in osmolality (Wood, 1999). C. glutamicum responds to a sudden osmotic upshift by the uptake of betaine, ectoine or proline (Morbach \& Krämer, $2003,2005)$. When these compunds are not available, $C$. glutamicum synthesizes compatible solutes such as proline and trehalose (Morbach \& Krämer, 2003, 2005). For trehalose synthesis, C. glutamicum possesses the OtsAB and the TreYZ pathway (Tzvetkov et al., 2003; Wolf et al., 2003). Absence of intracellular trehalose has been observed when both the OtsAB and the TreYZ pathways are 
inactivated in C. glutamicum (Tzvetkov et al., 2003; Wolf et al., 2003). The same phenotype has been observed when glycogen synthesis is abolished by disruption of the glycogen synthase gene glgA in C. glutamicum $\triangle$ ots $A B$ (Tzvetkov et al., 2003). Moreover, overexpression of the treYZ genes in C. glutamicum causes a decrease in the intracellular glycogen content (Carpinelli et al., 2006). All these findings indicate a link between trehalose formation through the TreYZ pathway and glycogen synthesis in the cells. We showed here that a shift to hyperosmotic growth conditions during the exponential-growth phase of $C$. glutamicum WT caused an immediate decrease in the intracellular glycogen content. In parallel to the decrease in glycogen content, an increase in the intracellular trehalose concentration took place. In contrast, the mutant strain $C$. glutamicum WT-ImX reacted with a complete cessation of growth on exposure to hyperosmotic conditions. Neither enzymically nor by TLC analysis could the formation of trehalose be observed in cell extracts of C. glutamicum WTImX. This corresponds to the same phenotype as $C$. glutamicum $\Delta$ treY after an upshift of osmolality (Wolf et al., 2003). Since the $g \lg X$ mutant contains glycogen, in principle, it should synthesize and contain all the intermediates of glycogen synthesis, including the linear chains of $\alpha$-1,4-glycosidic-linked glucose residues, the products of glycogen synthase (Tzvetkov et al., 2003), and the substrate for TreZ (Carpinelli et al., 2006). The fact that the mutant contains no trehalose thus indicates that mainly the maltodextrins derived from the GlgX activity in the course of glycogen degradation are used for trehalose synthesis via the TreYZ pathway, and not the maltodextrins derived from de novo synthesis through the action of ADPglucose pyrophosphorylase and glycogen synthase. In other words, the $\mathrm{GlgX}$ protein is essential for the accumulation of free maltodextrins, which in turn, are required for trehalose synthesis.

Wolf et al. (2003) have found that inactivation of the TreYZ pathway leads to a reduction in the intracellular trehalose content; however, in contrast to our glgX mutant, the growth of the $\Delta$ treY mutant after osmotic up-shift is not significantly impaired. Those authors explain this result by the dominant role of proline as a compatible solute under their experimental conditions. In view of this hypothesis, the growth impairment of C. glutamicum WTImX on exposure to hyperosmotic conditions is surprising, since proline synthesis should not be affected by $\operatorname{glg} X$ inactivation. In fact, the phenotype of $C$. glutamicum WTImX resembles that of the mutant deficient in both the TreYZ and OtsAB pathways (i.e. cytoplasmic trehalose and complete cessation of growth after hyperosmotic shock), and therefore we conclude that $g \lg X$ inactivation might also have an influence on the activity of the OtsAB pathway. In view of this hypothesis, it is interesting to note that in $E$. coli, immediately after hypersomotic shock, the uptake of glucose is drastically inhibited (Roth et al., 1985), which leads to a shortage of metabolites such as glucose 1phosphate, ADP-glucose and UDP-glucose. By analogy, metabolites derived from glucose might also become limiting in C. glutamicum after hyperosmotic shock. The pathways for trehalose synthesis require such metabolites either directly (OtsAB pathway, glucose 1-phosphate and UDP-glucose) or indirectly (de novo trehalose formation from glycogen or intermediates of glycogen synthesis). The formation of trehalose from maltodextrins derived from the degradation of intracellular glycogen by GlgX provides C. glutamicum with a mechanism for fast adaptation to hyperosmotic conditions, without uptake of carbohydrates.

\section{ACKNOWLEDEMENTS}

The authors thank P. Dangel for excellent technical assistance. The support of Degussa AG, Halle-Künsebeck, is gratefully acknowledged.

\section{REFERENCES}

Abad, M. C., Binderup, K., Rios-Steiner, J., Arni, R. K., Preiss, J. \& Geiger, J. H. (2002). The X-ray crystallographic structure of Escherichia coli branching enzyme. J Biol Chem 277, 42164-42170.

Alonso-Casajús, N., Dauvillée, D., Viale, A. M., Munoz, F. J., BarojaFernandez, E., Moran-Zorzano, M. T., Eydallin, G., Ball, S. \& Pozueto-Romero, J. (2006). Glycogen phosphorylase, the product of the $g \lg P$ gene, catalyzes glycogen breakdown by removing glucose units from the nonreducing ends in Escherichia coli. J Bacteriol 188, 5266-5272.

Altschul, S. F., Gish, W., Miller, W., Myers, E. W. \& Lipman, D. J. (1990). Basic local alignment search tool. J Mol Biol 215, 403-410.

Ball, S. G. \& Morell, M. K. (2003). From bacterial glycogen to starch: understanding the biogenesis of the plant starch granule. Annu Rev Plant Biol 54, 207-233.

Ballicora, M. A., Iglesias, A. A. \& Preiss, J. (2003). ADP-glucose pyrophosphorylase, a regulatory enzyme for bacterial glycogen synthesis. Microbiol Mol Biol Rev 67, 213-225.

Belanger, A. E. \& Hatfull, G. F. (1999). Exponential-phase glycogen recycling is essential for growth of Mycobacterium smegmatis. J Bacteriol 181, 6670-6678.

Buschiazzo, A., Ugalde, J. E., Guerin, M. E., Shepard, W., Ugalde, R. A. \& Alzari, P. M. (2004). Crystal structure of glycogen synthase: homologous enzymes catalyse glycogen synthesis and degradation. EMBO J 23, 3196-3205.

Carpinelli, J., Krämer, R. \& Agosin, E. (2006). Metabolic engineering of Corynebacterium glutamicum for trehalose overproduction: role of the TreYZ trehalose biosynthetic pathway. Appl Environ Microbiol 72, 1949-1955.

Cerdeno-Tarraga, A. M., Efstratiou, A., Dover, L. G., Holden, M. T. G., Pallen, M., Bentley, S. D., Besra, G. S., Churcher, C., James, K. D. \& other authors (2003). The complete genome sequence and analysis of Corynebacterium diphtheriae NCTC13129. Nucleic Acids Res 31, 65166523.

Cramer, A., Gerstmeir, R., Schaffer, S., Bott, M. \& Eikmanns, B. J. (2006). Identification of RamA, a novel LuxT-type transcriptional regulator of genes involved in acetate metabolism of Corynebacterium glutamicum. J Bacteriol 188, 2554-2567.

Dauvillée, D., Kinderf, I. S., Zhongyi, L., Kosar-Hashemi, B., Samuel, M. S., Rampling, L., Ball, S. \& Morell, M. K. (2005). Role of the Escherichia coli glgX gene in glycogen metabolism. J Bacteriol 187, 1465-1473. 
De Smet, K. A. L., Weston, A., Brown, I. N., Young, D. B. \& Robertson, B. D. (2000). Three pathways for trehalose biosynthesis in mycobacteria. Microbiology 146, 199-208.

Dippel, R., Bergmiller, T., Böhm, A. \& Boos, W. (2005). The maltodextrin system of Escherichia coli: glycogen-derived endogenous induction and osmoregulation. J Bacteriol 187, 8332-8339.

Eggeling, L. \& Bott, M. (2005). Handbook of Corynebacterium glutamicum. Boca Raton, FL: CRC Press.

Eikmanns, B. J., Metzger, M., Reinscheid, D., Kircher, M. \& Sahm, H. (1991). Amplification of three threonine biosynthesis genes in Corynebacterium glutamicum and its influence on carbon flux in different strains. Appl Microbiol Biotechnol 34, 617-622.

Eikmanns, B. J., Thum-Schmitz, N., Eggeling, L., Ludtke, K. U. \& Sahm, H. (1994). Nucleotide sequence, expression and transcriptional analysis of the Corynebacterium glutamicum gltA gene encoding citrate synthase. Microbiology 140, 1817-1828.

Fleischmann, R. D., Alland, D., Eisen, J. A., Carpenter, L., White, O., Peterson, J., DeBoy, R., Dodson, R., Gwinn, M. \& other authors (2002). Whole-genome comparison of Mycobacterium tuberculosis clinical and laboratory strains. J Bacteriol 184, 5479-5490.

Friedberg, F. \& Rhodes, C. (1986). Cloning and characterization of the beta-amylase gene from Bacillus polymyxa. J Bacteriol 165, 819-824.

Garnier, T., Eiglmeier, K., Camus, J.-C., Medina, N., Mansoor, H., Pryor, M., Duthoy, S., Grondin, S., Lacroix, C. \& other authors (2003). The complete genome of Mycobacterium bovis. Proc Natl Acad Sci U S A 100, 7877-7882.

Hanahan, D. (1983). Studies on transformation of Escherichia coli with plasmids. J Mol Biol 166, 557-580.

Horcajada, C., Guinovart, J. J., Fita, I. \& Ferrer, J. C. (2006). Crystal structure of an archaeal glycogen synthase. J Biol Chem 281, 2923-2931.

Iglesias, A. A. \& Preiss, J. (1992). Bacterial glycogen and plant starch biosynthesis. Biochem Educ 20, 196-203.

Ikawa, K., Araki, H., Tsujino, Y., Hyashi, Y., Igarashi, K., Hatada, Y., Hagihara, H., Ozawa, T., Ozaki, K. \& other authors (1998). Hyperexpression of the gene for a Bacillus $\alpha$-amylase in Bacillus subtilis cells: enzymatic properties and crystallization of the recombinant enzyme. Biosci Biotechnol Biochem 62, 1720-1725.

Jeanningros, R., Creuzet-Sigal, N., Frixon, C. \& Cattaneo, J. (1976). Purification and properties of a debranching enzyme from Escherichia coli. Biochim Biophys Acta 438, 186-199.

Kalinowski, J., Bathe, B., Bartels, D., Bischoff, M., Bott, M., Burkovski, A., Dusch, N., Eggeling, L., Eikmanns, B. J. \& other authors (2003). The complete Corynebacterium glutamicum ATCC 13032 genome sequence and its impact on the production of Laspartate-derived amino acids and vitamins. J Biotechnol 104, 5-25.

Laemmli, U. K. (1970). Cleavage of structural proteins during the assembly of the head of bacteriophage T4. Nature 227, 680-685.

Liebl, W. (2005). Corynebacterium taxonomy. In Handbook of Corynebacterium glutamicum, pp. 9-34. Edited by L. Eggeling \& M. Bott. Boca Raton, FL: CRC Press.

Manners, D. J. (1991). Recent developments in our understanding of glycogen structure. Carbohydr Polym 16, 37-82.

Miller, G. L. (1959). Use of dinitrosalicylic acid reagent for determination of reducing sugar. Anal Chem 31, 426-428.

Morbach, S. \& Krämer, R. (2003). Impact of transport processes in the osmotic response of Corynebacterium glutamicum. J Biotechnol 104, $69-75$.
Morbach, S. \& Krämer, R. (2005). Osmoregulation. In Handbook of Corynebacterium glutamicum, pp. 417-435. Edited by L. Eggeling \& M. Bott. Boca Raton, FL: CRC Press.

Nishio, Y., Nakamura, Y., Kawarabayasi, Y., Usuda, Y., Kimura, E., Sugimoto, S., Matasui, K., Yamagishi, A., Kikuchi, H. \& other authors (2003). Comparative genome sequence analysis of the amino acids replacements responsible for the thermostability of Corynebacterium efficiens. Genome Res 13, 1572-1579.

Padilla, L., Morbach, S., Krämer, R. \& Agosin, E. (2004). Impact of heterologous expression of Escherichia coli UDP-glucose pyrophosphorylase on trehalose and glycogen synthesis in Corynebacterium glutamicum. Appl Environ Microbiol 70, 3845-3854.

Preiss, J. (1984). Bacterial glycogen synthesis and its regulation. Annu Rev Microbiol 38, 419-458.

Preiss, J. (1996). ADPglucose pyrophosphorylase: basic science and applications in biotechnology. Biotechnol Annu Rev 2, 259-279.

Romeo, T., Kumar, A. \& Preiss, J. (1988). Analysis of the Escherichia coli glycogen gene cluster suggests that catabolic enzymes are encoded among biosynthetic genes. Gene 70, 363-376.

Roth, W. G., Leckie, M. P. \& Dietzler, D. N. (1985). Osmotic stress drastically inhibits active transport of carbohydrates by Escherichia coli. Biochem Biophys Res Commun 126, 434-441.

Sambrook, J. \& Russel, D. W. (2001). Molecular Cloning: a Laboratory Manual, 3rd edn. Cold Spring Harbor, NY: Cold Spring Harbor Laboratory.

Seibold, G. M., Dempf, S., Schreiner, J. \& Eikmanns, B. J. (2007). Glycogen formation in Corynebacterium glutamicum and role of ADPglucose pyrophosphorylase. Microbiology 153, 1275-1285.

Stackebrandt, E., Rainey, F. A. \& Ward-Rainey, N. L. (1997). Proposal for a new hierarchic classification system, Actinobacteria classis nov. Int J Syst Bacteriol 47, 479-491.

Tauch, A., Kaiser, O., Hain, T., Goesmann, A., Weisshaar, B., Albersmeier, A., Bekel, T., Bischoff, N., Brune, I. \& other authors (2005). Complete genome sequence and analysis of the multiresistant nosocomial pathogen Corynebacterium jeikeium K411, a lipid-requiring bacterium of the human skin flora. J Bacteriol 187, 4671-4682.

Thompson, J. D., Higgins, D. G. \& Gibson, T. J. (1994). CLUSTAL W: improving the sensitivity of progressive multiple sequence alignment through sequence weighting, position-specific gap penalties and weight matrix choice. Nucleic Acids Res 22, 4673-4680.

Tropis, M., Meniche, X., Wolf, A., Gebhardt, H., Strelkov, S., Chami, M., Schomburg, D., Krämer, R., Morbach, S. \& Daffé, M. (2005). The crucial role of trehalose and structurally related oligosaccharides in the biosynthesis and transfer of mycolic acids in corynebacterineae. $J$ Biol Chem 280, 26573-26585.

Tzvetkov, M., Klopprogge, C., Zelder, O. \& Liebl, W. (2003). Genetic dissection of trehalose biosynthesis in Corynebacterium glutamicum: inactivation of trehalose production leads to impaired growth and an altered cell wall composition. Microbiology 149, 1659-1673.

Wolf, A., Krämer, R. \& Morbach, S. (2003). Three pathways for trehalose metabolism in Corynebacterium glutamicum ATCC 13032 and their significance in response to osmotic stress. Mol Microbiol 49, 1119-1134.

Wood, J. M. (1999). Osmosensing by bacteria: signals and membranebased sensors. Microbiol Mol Biol Rev 63, 230-262.

Yeo, M. \& Chater, K. (2005). The interplay of glycogen metabolism and differentiation provides an insight into the developmental biology of Streptomyces coelicolor. Microbiology 151, 855-861.

Edited by: M. Hecker 